\title{
Some Comparisons between Radiation Effects in Polymeric and Biological Macromolecules
}

\author{
Dedicated to the Memory of the late Professor Ichiro Sakurada
}

A. Charlesby

Silverspring, Watchfield, Swindon, SN6 8TF, UK

(Received January 12, 1987)

\begin{abstract}
The fields of radiation effects in simple polymers and in biopolymers have been largely approached from very different aspects, although there are close analogies in many of these effects. Examples of these similarities in a number of cases include the influence of temperature, of oxygen, of certain additives, e.g., as radiation protectors, and of water with the indirect effect. Lessons learnt from the irradiation of polymers do not always appear to have been applied to more complex biological systems, and in particular the physical changes such as molecular configuration during irradiation, which can be of great importance on its subsequent behavior. The approximately similar $G$ values for radical production as between these two systems, and the considerable difference in doses required may be taken to imply that it is molecular weight and arrangement rather than the behavior of a specific chemical constituent which is a key element in explaining this difference, which is also found in polymers of similar chemical structure but of different molecular weight. One may also consider whether molecular crosslinking as well as main chain scission is the vital factor in determining subsequent vitality of a macromolecular biological system. Linking two macromolecules together at only one point anywhere would greatly limit their subsequent behavior, e.g., separation and duplication, and would not face the same problem as in naturally occurring radiation-induced reaction.
\end{abstract}

KEY WORDS Molecular Configuration / Scission / Crosslinks / Polymers and Biopolymers / Radiation Effects / Network Structure in Biopolymers /

Physical Arrangement of Macromolecules /

It has long been known that very close relationships exist between many chemical changes involved in radiobiology and those observed in the radiation chemistry of low molecular weight, relatively simple organic liquids or aqueous solutions. These have been subjected to an immense amount of study, and thereby open to radiobiologists a vast store of detailed and carefully analysed information on such model compounds. Consequently, there are many close interchanges between radiation chemists and radiobiologists.

The purely chemical reactions involved in the irradiation of long-chain polymers need not be inherently very different from those involved in the irradiation of lower molecular weight compounds of related chemical structure, but the association between polymer scientists and radiobiologists is far less direct. This is unfortunate, since, due to the influence of molecular arrangement, the analogies between simple polymeric macromolecules and biomolecules can be far closer. It would, therefore, appear useful to outline many of the affinities between the two, and to emphasize those aspects where the results obtained in the irradiation of polymers can be far more revealing than those derived from the study of low molecular compounds, usually in the liquid state. Of particular importance is the observation that a relatively very small energy input or dose can promote a drastic change in 
physical (and possibly biological) behavior, revealed in macroscopic changes apparently out of all proportion to the minute chemical modification.

\section{MOLECULAR CONFIGURATION}

The possibility of a simple linear polymer molecule existing in a variety of states, flexible, amorphous, rigid amorphous, oriented, in several crystalline states, or in a simultaneous combination thereof, depending on previous history and treatment, and each with its own radiation response, opens up further avenues for radiobiologists to explore, looking for direct analogies. One can certainly enquire how far molecular mobility and configuration, as distinct from minor chemical differences, influences biological response. As a simple example, consider a sample consisting of linear macro-molecules, of (weight) average molecular weight $10^{6}$, which crosslinks on irradiation with a $G$ (crosslink) value of 2 . On irradiation of up to a dose of 0.25 Mrads $(2.5 \mathrm{kGy})$, the initially linear molecules will include an increasing faction of star-shaped molecules, which might interfere with recrystallization rate; this increase in molecular weight would not be rendered apparent by any marked change in viscosity or flow properties, but would render the behavior of these molecules different from those of linear molecules of the same higher molecular weight. In biological molecules, this behavior may interfere with recrystallization or helical formation.

At higher doses, exceeding the critical gelation dose of $0.25 \mathrm{Mrad}$, there is a dramatic change in the properties of the macromolecule. The number of chemical bonds modified is as low as $10^{-6} \mathrm{molg}^{-1}$, a level of impurity far lower than would, in most cases, have a very significant influence on the pattern of behavior of low molecular weight compounds. In a macromolecular sample, this dose, and resultant few chemical modifications, produces a material which can no longer flow, but instead can undergo limited deformation under stress, which is recoverable, attempting to return to the configuration it held during irradiation. It therefore, acquires elastic properties. Also it can swell to an extent depending on dose and swelling agent and it is only partly soluble.

The expression for the minimum dose $r_{\mathrm{g}}$ (in Mrads) needed for this drastic change to be initiated is readily derived:

$$
r_{\mathrm{g}}=0.48 \times 10^{6} / \bar{M}_{w} G
$$

where $G$ is the crosslinking value, typically about $1-3$, but capable of reaching about 10 in certain structures, and $\bar{M}_{w}$ is the (weight) average molecular weight. Thus with $r_{\mathrm{g}} \sim 500$ rads, $\bar{M}_{w} \times 10^{9}$, a value comparable with that of many biopolymers. In such biological systems, the chemical changes can amount to only about 1 bond in $10^{8}$.

This crosslinking reaction may therefore explain why such minute depositions of energy, and correspondingly minute chemical changes can nevertheless produce a dramatic change in many important properties such as chain mobility, and restricted configurational changes, as well as impart a degree of memory influencing subsequent biological behavior.

These changes may occur at even lower doses if an adequate concentration of unsaturated groups is present. These allow a single radiation event to promote the crosslinking of a number of macromolecules by a chain reaction, somewhat akin to a polymerization reaction, and referred to as enhanced crosslinking. In such cases there is a very rapid increase in molecular weight, and a change in configuration, even at very low doses. Greater sensitivity to radiation may also appear when macromolecules are irradiated in an aqueous environment, when the radiolytic products of the water react with and promote reactions between adjacent macromolecules. This is the indirect effect so well-known in biological systems, and this has also been studied in great detail in model polymeric sys- 
tems. This greatly increased sensitivity is, however, more apparent than real, since if the total energy absorbed in the entire aqueous system, rather than that absorbed directly in the macromolecule itself is considered, no great enhancement need occur.

Of perhaps greater importance is the effect of macromolecular concentration. The lower the concentration (i.e., the further apart the macromolecules are) the lower is the dose needed to link them into a network structure. However, as the macromolecular concentration is lowered further (below about $0.5 \%$ typically) there is a sudden change in behavior. The dose needed for network formation increases rapidly; a point is soon reached at which no macroscopic gelled structure, swollen in water, is formed. This is due to competition between inter- and intramolecular links, and at these low concentrations the latter predominates. This leads to the formation of a series of highly crosslinked microgel fragments, in which each initial molecule (perhaps linear in structure) is increasingly converted towards an approximately spherical structure.

This shows clearly the effect of macromolecular concentration on radiation response, even if the purely radiation chemical changes are identical. It would be interesting to discover whether this marked dependance on concentration is also observed in purely biological systems.

Another aspect of this type of investigation concerns the effect of $\mathrm{pH}$ and similar parameters, which can influence the shape of the macromolecule during irradiation. This does not appear to have been studied in adequate detail. In solutions which encourage each individual macromolecule to fold back on itself, or take a more condensed configuration, there will be an enhanced promotion of such microgel, internally linked particles. At somewhat different solution conditions, which allow the molecules to open up, macrogel formation is favored. Thus a major differ- ence in physical and presumably biological behavior can be found, with little or no change in apparent radiation-chemical behavior.

In other polymers, the effect of radiation is to cause scissions in the main chain (degradation) with the formation of radicals at the points of fracture, which ultimately react and disappear, leaving a permanent site of scission of the main chain, a reduction in molecular weight and of course an increase in mobility. This behavior can also occur in the solid, crystalline or frozen state, and one may wonder why two highly reactive radicals, held for a long period in the closest proximity, do not react with each other to repair the fracture. One explanation is that many macromolecules which scission under radiation have large sidegroups attached on pairs to the backbone, and these produce a state of molecular strain for steric reasons. Once the chain is broken, the fracture allows the two fragments to separate. Some such behavior must of course occur in many bimolecules. As an example poly(methyl methacrylate), with $-\mathrm{CH}_{3}$ and $\mathrm{COOCH}_{3}$ sidegroup, scissions under radiation (main chain fracture) whereas poly(methyl acrylate), with $-\mathrm{H}$ and $\mathrm{COOCH}_{3}$ sidegroups crosslinks (preferential side chain fracture).

This basic pattern of behavior of polymer which scission under irradiation opens up a number of questions which must also occur in many biological systems. Radiation protection can be observed with very low concentration of additive, which would not be present at the point of scission. Two methods of protection can be envisaged - an immediate energy transfer within the frozen, immobile system to the additive molecule; and a longer-term repair mechanism, bridging the two broken chains. These two mechanisms could to a large extent be different from those encountered in macromolecules which prefer to crosslink under radiation. These varied mechanisms must also occur in biological systems, but may be ana- 
lyzed more readily in polymeric systems and then applied in radiobiology.

\section{TEMPERATURE DEPENDENCE}

Both biological and polymeric systems show a dependence on the temperature at which irradiation is carried out. Early radiobiological research results were explained on the basis of differences in biological behavior at various temperature. However, it was found that the temperature dependence of scission in irradiated polyisobutylene, over a range from $-200^{\circ} \mathrm{C}$ to $+100^{\circ} \mathrm{C}$, was similar to the inactivation of $E$. coli and of red cell catalase, and at least in these systems no specific biological explanation is needed. It may be presumed that for many other biological systems the temperature effect of radiation is that found in other and simpler macromolecules, and arises from some purely radiation chemistry phenomenon, or from increased molecular mobility.

To avoid off-odors or tastes the radiation sterilization of foodstuffs is carried out at low temperature. One can see that this will result in smaller radiation change and damage. However, if this parallel between the temperature dependence for food, for polymer and for undersirable bacteria persists, no advantage is gained-the same effect could be achieved at room temperature by a smaller dose (and of course a smaller degree of sterilization). The explanation appears to be that food contains a large proportion of water, so that the radiation effect is greatly enhanced by the indirect effect. This indirect effect is greatly diminished at low temperature, when the water is converted to ice. For the bacteria it may be surmised that the water content and hence indirect effect are much smaller, and there is a much smaller loss in effectiveness when irradiation is carried out below the icing temperature.

Another major difference in radiation response as between high and low molecular weight compound arises from the possibility of several states at the same temperature. Thus crystalline regions may exist in stable equilibrium with amorphous phase. Although the initial energy absorption is similar in both these two components, the effect may be very different. The radicals or ions formed in the crystalline regions may be unable to react there, but be capable of migrating in some way (to be discussed separately) to the crystalline surface where because of increased chain mobility, reaction takes place preferentially. One can therefore envisage at least three distinct concentrations and possibly types of radiation effect-within the crystalline region, on its surface, and throughout the amorphous bulk. Moreover the relative fractions of these components may also depend on previous history. Thus considerable differences can occur in radiation response of a macromolecular material at a single temperature, which would not be expected from the radiation chemistry of a comparable low molecular weight compound.

So far most of the researches in this field have been concerned with polymers such as polyethylene, which below the melting point consists of both crystalline and flexible amorphous components. One may also study the effects of state on crosslinking amorphous polymer below the glass temperature, when the individual chains are rigid, and intermolecular reactions are inhibited. The amount of research in such materials is far more limited. Does the crosslinking reaction for example occur in the rigid amorphous state, or do the radicals combine only when the specimen is warmed and the macromolecules can move?

One may also consider the behavior of radical macromolecules in polymers such as poly(methyl methacrylate), which are amorphous at all temperatures, and which suffer main chain (and side chain) scission even when irradiated at temperatures below the glass temperature. One might imagine that when the two adjacent radical ends at the newly-formed point of scission are kept in very close proximity, there would be a great probability of 
rejoining when warmed up. At much higher temperature of irradiation, when the two fractured radical chain ends can immediately move apart, one would expect a much higher scission probability. In fact the temperature difference below and above the glass temperature is surprisingly small.

\section{ENERGY TRANSFER AND RADIATION PROTECTION}

Even in the early history of radiation chemistry of organic liquids, the degree of protection offered by relatively small concentrations of aromatics was well documented. The explanation widely accepted is that the energy absorbed in the entire specimen is largely transferred to the aromatic ring, where it can be dissipated into the excited states, with little permanent change in chemical structure. This type of radiation protection is also found in polymeric molecules containing aromatic groups notably in polystyrene, where the $G$ value for crosslinking is as low as 0.1 as compared with about 1.5 for polyethylene. To be strictly comparable both measurements should be for molecules with the same mobility, i.e., both with no crystalline phase and either above or below the glass temperature. However, the $G$ value for initiation of polymerization of the styrene monomer, with the same degree of aromaticity does not show such a considerable protective effect. Other polymeric system containing aromatic groups are also more radiation resistant, and it is possible to estimate the approximate range over which this protection effect is operative at about four carbon atoms. Obviously this mechanism of radiation protection can only be effective during the irradiation time itself.

Aromatic or other forms of unsaturation may be effective in other ways, e.g., by acting as a $\mathrm{H}$ sink, reducing the extent of reactions such as crosslinking in which $\mathrm{H}$ plays a role. The very slow decay of radicals in crystalline or solid amorphous polymers, as seen by ESR is known to depend on $\mathrm{H}_{2}$ pressure, so that the addition/abstraction reactions must be of low probability, allowing even a very small concentration of additive to be effective in reducing the effect, even in a macromolecular solid with very little molecular motion.

\section{ANTIOXIDANTS AND ANTIRADS}

The deterioration with time of mechanical properties of polymers in wide-spread use is a continuing problem on polymer science, and is largely ascribed to polymer reaction with oxygen. It is therefore directly relevant to the behavior of irradiated polymeric and biological macromolecules, where oxygen can react readily with radiation-induced radicals and modify subsequent reactions. A prominent example is the deterioration in properties of irradiated polypropylene, widely used in radiation-sterilized medical products; here a chain reaction of polymer radicals with oxygen is involved, lasting a period of months, and ultimately causing main chain scission.

To obviate, or at least reduce the effect, additives are incorporated, which by reacting with reactive groups, reduce the chance of their reacting with oxygen. These antioxidants are only present in low concentration in a relatively rigid matrix, and it is necessary to envisage a mechanism by which the macromolecular radical can meet the additive molecule. The same problem of course arises in accounting for crosslinking.

By reacting with these radicals and thereby stabilizing them, the effect of the radiation dose is reduced, and these antioxidants are therefore considered as radiation protectors or antirads. For example in some typical polymer, the rate of loss of antioxidants during the radiation process can greatly exceed the energy they absorb directly, and some mechanisms of transfer of energy or of reactivity to the additive is needed to account for this behavior.

One must consider separately the reactions 
involving oxygen within a crystalline or rigid amorphous phase, and those in flexible molecular phase. In the former it is necessary to determine whether the trapped radicals migrate very slowly to the surface or into the more flexible molecular regions, where oxygen can readily penetrate, or whether it is the oxygen which diffuses very slowly into the crystalline regions, e.g., via a series of defects.

Many of the antioxidants comprise aromatic residues, which can protect by energy transfer, $\mathrm{H}$ capture, etc. These mechanisms are not open to the same additive present in a nonirradiated polymer, and it therefore becomes possible to determine the mechanism of protection by finding whether an additive is equally effective as an antioxidant in a non-irradiation situation.

If the eventual radiation-chemical product is due to the combination of two radicals on separate molecules, this will be in direct competition with a reaction with oxygen or with the antioxidant, increasing the dose needed. This competition can be determined by (a) the initial concentration of oxygen molecules, which can react with, and eliminate radicals produced in an irradiated polymer by an initial dose of perhaps one-quarter megarad and (b) the subsequent diffusion of oxygen into the crystalline regions. Thus we may find a radiation effect such as cross-linking apparently dependent on initial dose and dose rate which determines the relative importance of

$$
\begin{aligned}
& \mathrm{R} \cdot+\mathrm{R} \cdot \rightarrow \mathrm{R}_{2} \text { or } \\
& \mathrm{R} \cdot+\mathrm{O}_{2} \rightarrow \mathrm{RO}_{2} \quad \text { etc. }
\end{aligned}
$$

In general the ability of an additive, acting as an antioxidant or protector will depend not only on chemical structure, but on the state of the macromolecule being irradiated, e.g., crystal size and mobility.

\section{RADICAL MOBILITY}

The difference between the life time of identical radicals in the crystalline and amorphous flexible state of a polymer can be very considerable, e.g., in polyethylene. Indeed there is some doubt as to how the radicals trapped within a crystal disappear. Do they migrate to the crystal surface, where they react rapidly in the far more flexible phase with radicals on other macromolecular chains, or with oxygen, etc.? And if so what is the mechanism of this transfer? One suggestion is by a series of $\mathrm{H}$ transfers from adjacent bonds in which case the decay will be very temperature dependent even at low temperature (as is indeed observed). An alternative possibility is the reaction of radicals with free hydrogen, produced during the irradiation process itself. By a series of addition and abstraction reactions with $\mathrm{H}$ and $\mathrm{H}_{2}$ the radical can move within the crystal lattice until it meets an adjacent partner to form a crosslink even within the lattice, or onto the surface.

$$
\begin{gathered}
\mathrm{R}_{\mathrm{i}}+\mathrm{H}_{2} \rightarrow \mathrm{RH}+\mathrm{H} \\
\mathrm{R}_{2} \mathrm{H}+\mathrm{H} \rightarrow \mathrm{R}_{2}+\mathrm{H}_{2} \\
\mathrm{R}_{3}+\mathrm{H}_{2} \rightarrow \mathrm{R}_{3} \mathrm{H}+\mathrm{H} \\
\text { etc. } \\
\mathrm{R}_{n}+\mathrm{R}_{m} \rightarrow \mathrm{R}_{n} \mathrm{R}_{m}
\end{gathered}
$$

The fact that one or other of these reactions is of extremely low probability argues in favor of this hypothesis since the radical decay rate is in fact very slow. Of greater importance to this hypothesis is the observation that the radical decay rate increases with increasing $\mathrm{H}_{2}$ pressure. By this mechanism radicals can move about the crystal (not by a series of transitions between neighboring sites) thus additives in very low concentration capable of reacting with the polymer radicals, or with the $\mathrm{H}_{\text {or }} \mathrm{H}_{2}$ can greatly influence and reduce or delay the radiation effect-one form of radiation protection. 


\section{RADIATION MECHANISMS IN CROSSLINKING}

The discovery that macromolecules can be crosslinked by radiation has led to the development of a large-scale radiation industry applied to polymers, amounting to well over $\$ 10^{9}$ per annum, and increasing rapidly. In spite of the considerable research effort which the production can justify, no general agreement has been reached as to the mechanisms involved. Since the same crosslinking behavior must occur in some biological systems, greatly affecting their properties, its understanding should prove of great value. In early work it was indeed suggested that cancer may involve some form of crosslinking - it can be produced by radiation and by chemical crosslinking agents, it can be modified by oxygen and by radical inhibitors, and might be expected to show the same temperature dependence.

From the considerable amount of research information which has appeared in over thirty years, some important conclusions emerge.

(1) $G$ (crosslink) depends on physical state, being very different in crystalline and flexible amorphous regions. It has not yet been established how the radicals formed in the crystalline state disappear eventually; a $\mathrm{H} / \mathrm{H}_{2}$ addition-abstraction is involved, but is probably not sufficient in itself to explain all the radical changes and migration.

(2) In due course the radicals formed in the crystals disappear, but not all by reacting with each other. This is seen for example in the very different degree of crosslinking in polyethylenes of different degrees of crystallinity.

(3) Crosslinking can be reduced, but not completely eliminated by the presence of certain additives. This and other data point to the existence of two reaction mechanisms, one immediate which cannot be prevented, and a second slower one probably involving radical migration, against which radiation protection can be effective: An ionic reaction may be involved in the former.

(4) Some form of radical mobility must be envisaged to allow pairs to migrate into close proximity and to react at relatively low temperature. Radiation protection presumably occurs during this migration.

One must assume that similar behavior take place in biological macromolecules, and the problem is to determine its biological effect. One suggestion is that some form of mutation, of a drastic type, takes place when a molecular configuration is to some extent frozen by such a linkage, introducing a permanent loop into a long-chain structure. In mobile macromolecules such loops would in any case occur but these would be of a temporary, dynamic nature due to chain entanglements or associations within or between adjacent macromolecules. Molecular chain scission is another likely event, occurring at relatively low doses, but having a potentially large biological effect.

\section{IONIC REACTIONS}

In irradiated gases, ionic reactions play a major role, whereas in liquid or solid organics, the major effects arise from radical reactions, ultimately due to excited states. This difference is understandable since in the condensed state, the electron loses its excess energy rapidly and is very unlikely to escape the electric field of the parent ion. It is either recaptured immediately or trapped in its vicinity from which it returns subsequently either by thermal detrapping or tunnelling. Ionic polymerization, delayed thermoluminescence and induced conductivity can be a consequence of irradiation at low temperature. Since trace water greatly inhibits these reactions, one would expect such ionic or electron reactions to occur primarily in crystalline regions. Solid state reactions such as polymerization may indeed occur more rapidly than in the liquid state, and can be greatly influenced by additives capable of trapping the electron, as well as by molecular 
orientation in a regular structure.

Release of the electron and recombination lead to emission of light but not necessarily from the originally ionised species. Thus some mechanism of energy or charge transfer can operate, some considerable time after the completion of radiation. It is not known how far such reactions can be of biological significance.

\section{PULSED NMR AND MOLECULAR CONFIGURATION}

The use of NMR techniques to study chemical structure is a very well-established method of analysis. The use of pulsed NMR is very different, and recent work has shown that it can be used to analyze macromolecular configurations-molecular weight and mobility, network fraction and crosslink and entanglement density. Insofar as they are modified by radiation, its effect on macromolecules can be readily followed. The influence of radiation protection, fillers and reinforcing agents, crystallisation conditions, etc., can also be traced. Only a few tentative measurements along these lines have yet been made with corresponding biomolecules.

\section{RETENTION OF MOLECULAR ARRANGEMENT IN THE LIQUID STATE}

Recently considerable evidence has been obtained, most convincingly by pulsed NMR, showing that a high molecular weight polymer, in the liquid state (e.g., well above the glass temperature or the crystal melting temperature) can still retain some memory of its structure in its previous state. In this respect liquid structure in macromolecular materials means something quite different from in liquid molecules of low molecular weight. This behavior is ascribed to the formation of so-called entanglements-associations would perhaps be a better term, since their precise nature is not yet fully agreed. These entanglements are dynamic in nature, disappearing and reforming. If their number is sufficient to provide at least one entangled unit per molecule, a dynamic network is formed, retaining some degree of structure in the liquid state. Under stress it will deform at a rate which depends on the entanglement density and lifetime. This accounts for the well-known rheological behavior of polymers, which can show a combination of viscous and elastic flow. To completely separate these macromolecules it is necessary to greatly dilute them in a solvent, whereupon the "memory" disappears. The lifetime of this memory depends on entanglement density per molecule and temperature.

In a typical polymer the entanglement density is of the order of one entanglement per molecular weight of $3 \times 10^{4}$, but this must depend on structure (chain flexibility) and especially on temperature, and is greatly increased in solvents which separate entangled molecules. A high molecular weight degrading polymer, initially shows this "memory" effect, but when radiation reduces the molecular weight below this figure, the whole "fluid" system undergoes a very great change in properties, from a flexible network system, to one which is a true flowing liquid.

This considerable transition in mobility is not primarily due to any radiation chemical change, but merely to a change in molecular weight and hence degree of entanglement and mobility. One can therefore ask whether any biological behavior is similarly influenced by the degree of molecular entanglement and memory of prior states, and whether any observed changes due to radiation may result from its effect on these macromolecular properties, as demonstrated in simple polymer macromolecules.

\section{CONCLUSIONS}

The purpose of this contribution is to show in general terms that in macromolecular 
systems-polymeric or biological-great anisms of transfer of reactive groups and changes in molecular configuration can result radiation protection in immobile or mobile from very small doses and chemical modifi- components. It would be difficult to obtain cations. In this respect studies of radiation corresponding data from the radiation chemeffect in simple polymers can provide valuable istry of low molecular weight compounds, models for such matters as the influence of where long-range configuration plays little molecular arrangement and mobility, mech- part. 